\title{
Body Mass Index Variation by Even a Single Meal!
}

\author{
Seyyed-Mohsen Hosseininejad ${ }^{1}$, Fateme-Sadat Hosseininejad ${ }^{2}$, Seyyed-Mehran Hosseini ${ }^{3 *}$ \\ ${ }^{1}$ Student of Medicine, Student Research Committee, Golestan University of Medical sciences, Gorgan, Iran \\ ${ }^{2}$ Student Research Committee, Shahrood University of Medical sciences, Shahrood, Iran \\ ${ }^{3}$ Associate professor of Physiology, Department of Physiology, Golestan University of Medical sciences, Gorgan, Iran
}

Received: December 12, 2017; Published: December 18, 2017

*Corresponding author: Seyyed Mehran Hosseini,Associate professor of Physiology, Department of Physiology, Golestan University of Medical sciences, Gorgan, Iran

Abbreviations: WHO: World Health Organization; WC: Waist Circumference; BMI: Body Mass Index; GOUMS: Golestan University of Medical Sciences; ASIS: Anterior Superior Iliac Spine

\section{Opinion}

The prevalence of obesity has immensely increased around the world in both adults and children. In fact the World Health Organization (WHO) estimates that at least 1 billion people are overweight, and three hundred million of these are obese [1]. The rising prevalence of obesity merits the need for accurate methods of assessing adiposity. There are now, however, many measures of obesity, anthropometrics and otherwise [2]. Evidence from recent epidemiological studies has yielded the advocacy of WC (waist circumference) and BMI (Body Mass Index) as easy-to-use, low-cost, yet reliable measures of obesity $[3,4]$. As it is clear, BMI provides a simple numerical scale for body status often applied in population studies. BMI in medical literature is reported as variable (dependent or non-dependent) and also used for descriptions and classification of groups or populations. However, some studies apply BMI and WC based on self-reported body weight and height without any valid protocol; this subjective approach may potentially lead to inaccurate data [5]. So here, as very small part of the big world of researches, I am writing this letter to brief those involved in researches about my own study in which body weight changes following a single meal session could affect BMI and WC values.

We conducted a cross-sectional study on 120 students of Golestan University of Medical Sciences (GOUMS) in 2015; all participants were healthy college students and their mean ages were $19 \pm 3$ years. Body weight and waist circumference were measured before and after a meal according to the standard guideline of World Health Organization [6]. BMI was calculated as Mass (kg)/ [Height (m)] ${ }^{2}$ and was compared by Paired Student t-test. We noticed that there were statistically significant differences between body weights, WC and BMI values before and after a meal $(\mathrm{P}<0.05)$. Because having some food could increase body weight and immediately measurement of BMI and even WC thereafter make these indices be overestimated. Besides we observed that where to put the plastic tape on the body to measure the WC is crucial to the correct outcome; we performed WC measurement on three different point of the body: below the anterior superior iliac spine (ASIS), on and above it and three different values were obtained.

What we found in most studies applying BMI and other anthropometric indices, fasting condition for measurements of body weight might be ignored or at least not clearly reported while BMI calculation because it is supposed that its effect is very small and hardly can lead to the BMI categorical states bias. We suggest that the site of measurement and the time since last meal should be standardized for the development of a protocol of BMI and WC measurement; changes, although being slight, in cut-off points can make BMI and WC values move into another category; may cause analysis mismatch and remarkable changes in the final results which lead to interpretation bias because such anthropometric indices are generally used in population studies; so very little changes may produce very profound consequences thus inconclusive and wrong interpretations.

\section{References}

1. Rothman KJ (2008) BMI-related errors in the measurement of obesity. Int J Obes 32: 556-559.

2. Ejike, Ijeh (2012) Obesity in young-adult Nigerians: variations in prevalence determined by anthropometry and bioelectrical impedance analysis, and the development of $\%$ body fat prediction equations. International Archives of Medicine 5: 22.

3. Cheng TO (2004) Waist circumference versus body mass index in risk prediction of coronary heart disease: comparing apples and oranges. J Intern Med 255(6): 690-691. 
4. Wang J (2003) Waist circumference: a simple, inexpensive, and reliable tool that should be included as part of physical examinations in the doctor's office. Am J ClinNutr 78(5): 902-903.

5. Manfred Stommel, Charlotte AS (2009) Accuracy and usefulness of BMI measures based on selfreported weight and height: findings from the NHANES \& NHIS 2001-2006. BMC Public Health 9: 421.
6. Obesity: preventing and managing the global epidemic Report of a WHO consultation. (2000) World Health Organ Tech Rep Ser 894: 1-253.

$\begin{array}{ll}\text { BIOMEDICAL } & \text { Assets of Publishing with us } \\ \text { RESEARCHES } & \text { - Global archiving of articles } \\ & \text { - Immediate, unrestricted online access } \\ & \text { - Rigorous Peer Review Process } \\ \end{array}$

